\title{
In-Situ Orientation Imaging of Recrystallization and Grain Growth in OFHC Copper
}

\author{
M.M. Nowell,* S.I. Wright,* and J.O. Carpenter* \\ * EDAX-TSL, 392 E 12300 S Suite H, Draper, UT 84020 USA
}

The automated analysis of Electron Backscatter Diffraction (EBSD) patterns to determine the spatial distribution of crystallographic orientations is often termed orientation imaging, or orientation imaging microscopy. This technique has become well accepted and been widely used to characterize deformed microstructures. Recent advanced in data collection rates also make it a useful tool for observing dynamic changes in microstructure in-situ when used in conjunction with a heating and/or deformation (tensile or bending for example) stage. This work focuses on the in-situ characterization of recrystallization and grain growth in oxygen free high-conductivity (OFHC) copper using a heating stage designed for orientation imaging.

Samples from OFHC copper were deformed via equal channel angular extrusion (ECAE or ECAP) by a four or eight pass processing route. $10 \mathrm{~mm} \times 5 \mathrm{~mm} \times 2 \mathrm{~mm}$ sections were then removed from the center portion of each billet and prepared for orientation mapping with care to minimize any heat input through preparation. Single crystal copper crystals strained to approximately $10 \%$ were also prepared.

Samples were heated in-situ to a surface temperature of approximately $150^{\circ} \mathrm{C}$. Thermocouples were positioned to measure both the surface and heater temperatures. Care was taken to reach the desired heating temperature as quickly as possible without overheating the samples. Orientation maps were taken prior to heating, and then taken serially after a short initial period of specimen drift as the heating was initiated. Data collection rates varied from 70 to 250 indexed patterns per second. As data collection speed capabilities increased, the step size was held constant but the area of analysis was increased to improve sampling statistics. Analysis areas were selected to maintain a time per scan of 3-5 minutes.

Figure 1 shows orientation maps taken during the recrystallization process. Annealing twins are clearly developed during recrystallization. Figure 2 shows a Local Orientation Spread map ( ${ }^{\text {rd }}$ neighbor kernel) of three sequential scans at the point where recrystallization is first observed. This map type can be used to identify the recrystallized areas, which appear blue. Figure 3 shows the same scans where the recrystallized orientations are shown in red (first observed) and blue (second observed). Note than in the 32.5 minute scan two distinct nucleation sites of the same orientation are present, but by the next scan they have coalesced together. Three dimensional orientation images have been collected by automated serial sectioning using a Focused Ion Beam dual beam system to investigate the microstructure at the initial stages of recrystallization to examine the shape of the recrystallized nuclei.

\section{References}

[1] D.P. Field et al., Acta Materialia 55 (2007) 4233.

[2] M.M. Nowell et al., Materials Science Forum 467-470 (2004) 1401.

[3] M.M. Nowell et al., Microsc. Microanal. 11 (Suppl. 2) (2005) 1494. 

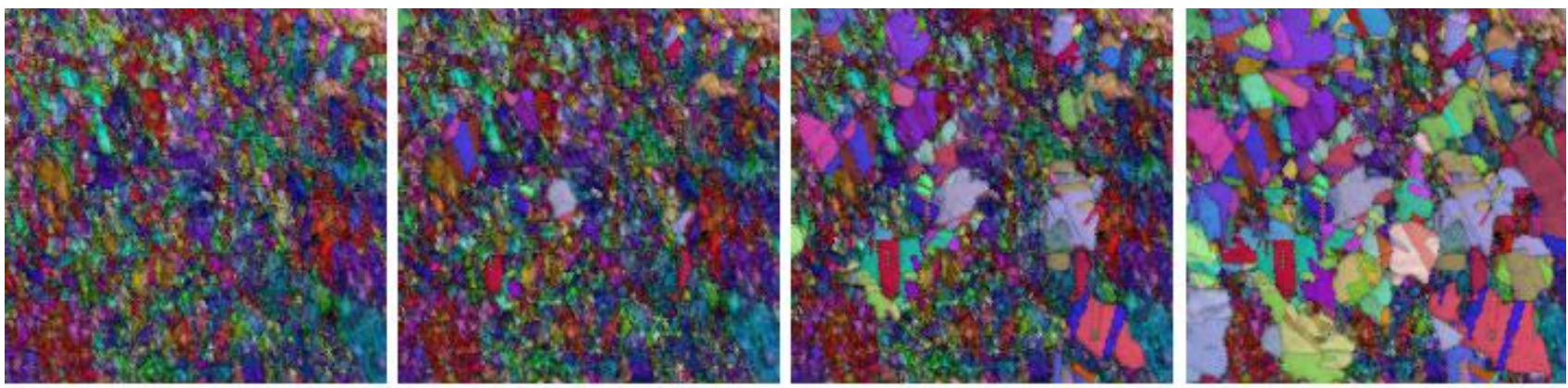

\section{$10 \mu \mathrm{m}$}

FIG. 1. Orientation maps at selected stages of recrystallization

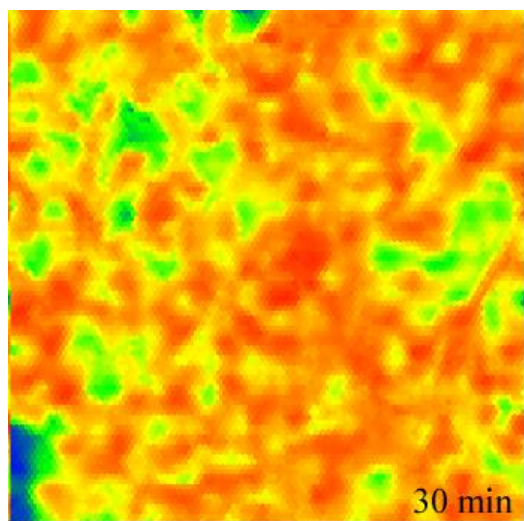

$5 \mu \mathrm{m}$

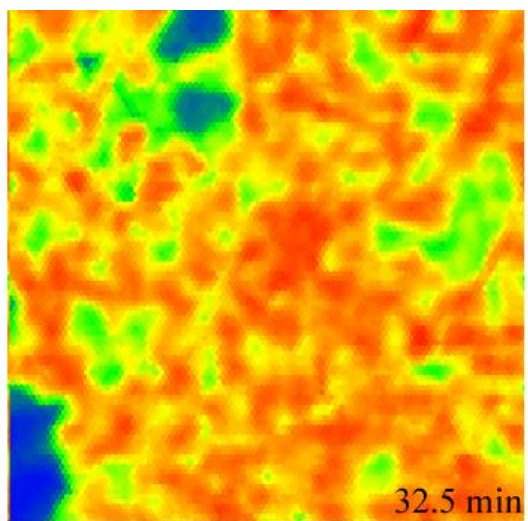

$32.5 \mathrm{~min}$

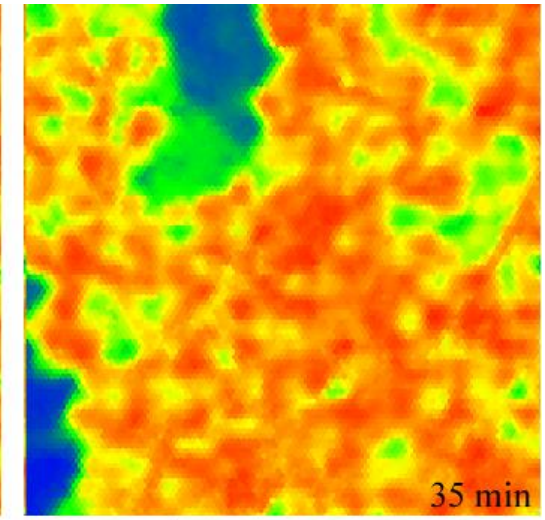

$0^{\circ}$ $3.5^{\circ}$

FIG. 2. Local Orientation Spread maps showing the regions of initial recrystallization

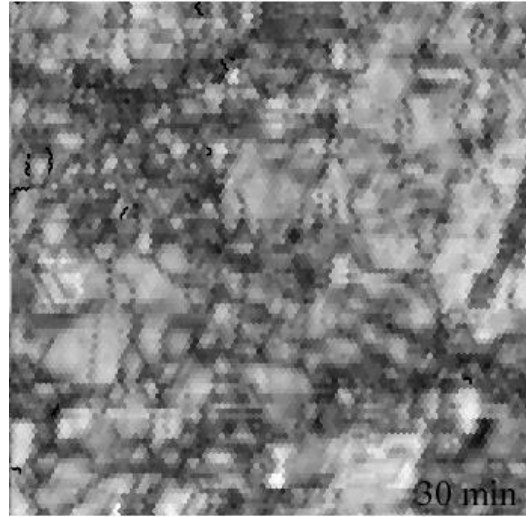

$5 \mu \mathrm{m}$
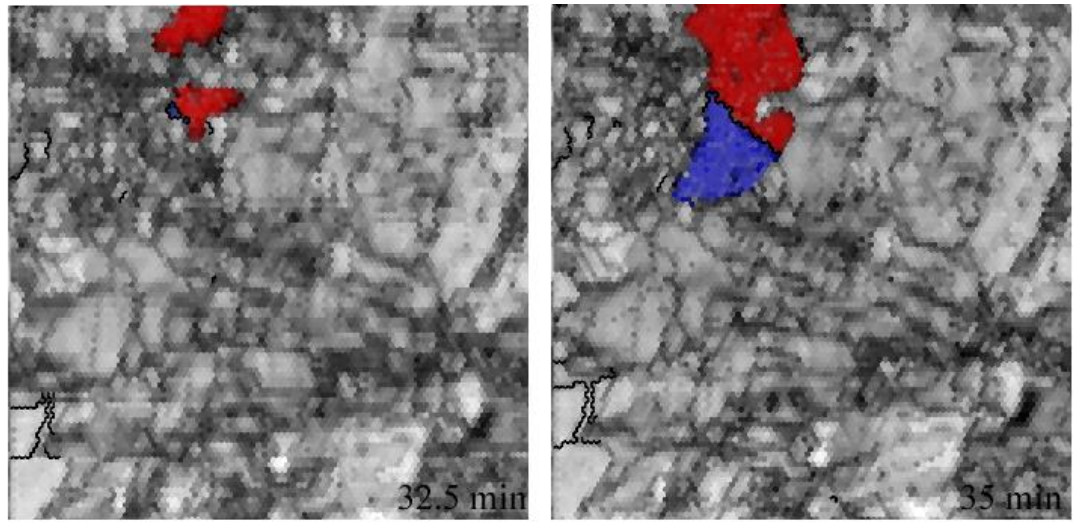

FIG. 3. Image quality maps with the first (red) and second (blue) recrystallized orientations colored. Twin boundaries are shown in black. Note the coalescence of the first orientation between the 32.5 and 35 minute scans. 